\title{
British Isles Lupus Assessment Group Index
}

National Cancer Institute

\section{Source}

National Cancer Institute. British Isles Lupus Assessment Group Index. NCI Thesaurus.

Code C121352.

An organ-specific assessment intended as a clinical decision tool. It evaluates specific manifestations over the previous four weeks in a total of eight organs systems, nine in the revised Index: constitutional, mucocutaneous, neuropsychiatric, musculoskeletal, cardiorespiratory, gastrointestinal, ophthalmic, renal, and hematological. Activity in each organ system is scored from most active disease to no previous activity. 